\title{
Orientation Insensitive Power Transfer by Magnetic Resonance for Mobile Devices
}

\author{
Olutola Jonah, Student Member, IEEE, Stavros V. Georgakopoulos, Senior Member, IEEE and \\ Manos M. Tentzeris, Fellow, IEEE
}

\begin{abstract}
The efficiency of wireless power transfer (WPT) from an orientation insensitive system to a mobile device by strongly coupled magnetic resonance (SCMR) is reported here. This paper compares an optimal loop-based design in standard SCMR systems with misalignment insensitive system (3D and 3loop structure), which exhibits higher efficiency than typical SCMR devices in several directions in a sphere.
\end{abstract}

Index Terms - Misalignment insensitive, mobile devices, magnetic resonance.

\section{INTRODUCTION}

$\mathrm{W}$ IRELESS power transfer methods have been utilized on several occasions in the past for various applications. An efficient orientation insensitive wireless power transfer mechanism is important for mobile devices because of the need to move around without power cables. Wireless power transfer has been implemented using near-field coupling in several applications such as RFID tags and implanted medical devices (IMDs) [1], [2]. In addition, some inductive coupling methods have exhibited high power transfer efficiencies $(>$ $90 \%)$ for very short distances $(1-3 \mathrm{~cm})$ [3]. But the efficiency of this method drops significantly for longer distance since it decays as $1 / \mathrm{r}^{6}$ [4], [5].

The SCMR method is a near field wireless mid-range power transfer technique $(10-300 \mathrm{~cm})$ [6-11]. The SCMR method achieved $40 \%$ wireless efficiency in the air for a distance of 2 $m$ with a single receiver [6]. Also, the SCMR technique was used to concurrently power multiple devices in the air, and has achieved a $60 \%$ at a distance of $2 \mathrm{~m}$ [7]. Previous work has shown that SCMR provides wireless power transfer efficiencies that are significantly larger than efficiencies of conventional inductive coupling methods [6], [7], [12]. In order for SCMR to achieve high efficiency, it requires that the transmitting and receiving elements (typically loops or coils) are designed so that they resonate at the desired operating frequency that must coincide with the frequency of where the elements exhibit maximum Q-factor. This paper focuses on the design of wireless powering systems based on SCMR method insensitive to misalignment, and hence improving power transfer efficiency in several directions. This is critical for efficient power transfer via SCMR because of its high sensitivity to misalignment.

In [13] an optimization technique to improve the efficiency of a WPT system in a lateral misalignment scenario was presented, in which demonstrated $48.4 \%$ efficiency was achieved with an adaptive matching network, but did not provide any solution for angular and rotational misalignment. The authors of [14] introduced analytical derivation for nearfield power transfer efficiency of loosely coupled inductive links with lateral and angular coil misalignment for three different coil geometries and scenarios, however this did not provide any solution for lateral, angular and rotational misalignment. In [15] showed the effect of changing of coil separation distance, lateral misalignment and angular misalignment, and also simultaneous data communications were demonstrated, this also did not furnish solutions for lateral, angular and rotational misalignment. In addition [16] presented an analytical and finite element method (FEM) analysis of misalignment effects on the WPT and in [17] the focus was on analyzing and optimizing of the energy transfer for inductive coupled system, with emphasis on misalignment analysis. Even though some of the papers above analyzed both lateral and angular misalignment, none proposed solution to the critical sensitivity exhibited by the SCMR system from lateral, angular and rotational misalignment effects in extreme misalignment conditions, this paper will demonstrate an orientation insensitive system that exhibit high efficiency (above 60\%) in several directions than standard SCMR.

\section{SCMR COIL MISALIGNMENTS}

The orientation and alignment of SCMR devices are critical in the design of efficient systems. A standard SCMR system exhibit high sensitivity to lateral, azimuth and angular misalignment. The rate of power transfer and losses across the inductive link changes as the alignments are altered, in this paper some of these are evaluated with the following form of misalignment:

a. Angular elevation misalignment

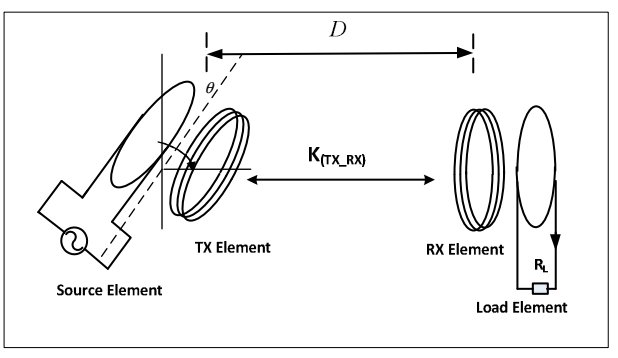

Fig. 1. SCMR power transfer system with angular elevation misalignment angles, $\theta . K_{T X} R X$ and $D$ are coupling and distance between TX and RX. 
In this case, the RX or TX (load or source elements) elements rotate in the elevation plane from $\theta=0^{\circ}$ to $90^{\circ}$ from the horizontal to the vertical plane, while the other device is unchanged, as shown in Fig. 1.

b. Angular azimuth misalignment - In this case, the RX or TX elements (with load or source elements) rotate on its axis from $\varphi=0^{\circ}$ to $360^{\circ}$ in the azimuth, while the other element is fixed, this is as shown in Fig. 2. The simulation and measurement result of the SCMR system with the above orientations are shown in the following section.

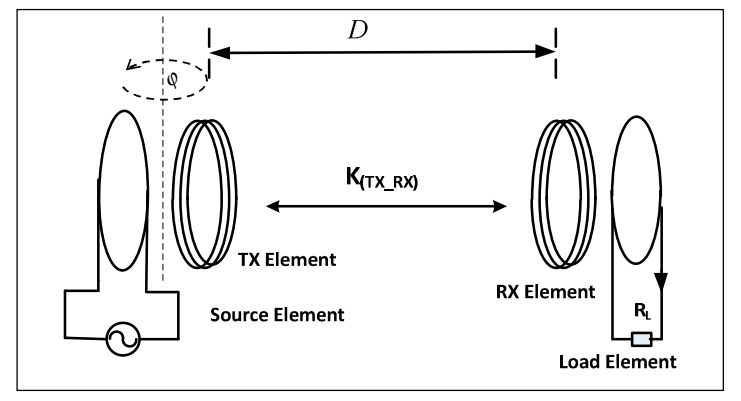

Fig.

2. SCMR power transfer system with angular misalignment angles, $\varphi . K_{T X \_} R X$ and $D$ are coupling coefficient and distance between TX and RX element.

c. Rotational misalignment - In this case, the RX or TX elements (with load or source elements) are rotated around the fixed elements from $\alpha=0^{\circ}$ to $360^{\circ}$ as shown in Fig.3.

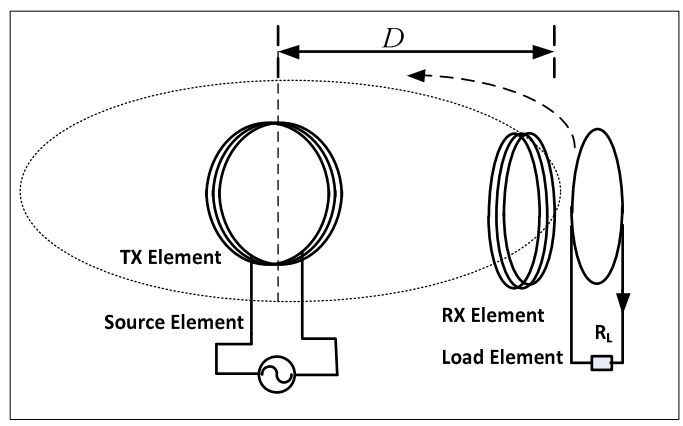

Fig. 3. SCMR power transfer system with misalignment angle $\alpha$ and $D$, the the distance between TX and RX element.

\section{OPTIMAL PARAMETERS OF SCMR SYSTEM}

SCMR requires that both the RX and TX elements exhibit maximum Q-factor at their resonant frequency $f_{r}$, in order to achieve maximum wireless efficiency. The frequency, $f_{r}$, is calculated from [18]:

$$
f_{r}=\frac{1}{2 \pi \sqrt{L C}}
$$

where $L$ and $C$ are the the self-inductance and resonating capacitance. The other basic parameters of the loops are $R_{\text {rad }}$ and $R_{\text {ohm }}$ which are the radiation and ohmic resistance of the loop, these three parameters are given by [18]

$$
\begin{aligned}
& L=\mu_{o} r\left[\ln \left(\frac{8 r}{r_{c}}\right)-2\right] \\
& R_{\text {rad }}=(\pi / 6) \eta_{o}\left(2 \pi f_{r} r / c\right)^{4}
\end{aligned}
$$

$$
R_{o h m}=\left(\sqrt{\mu_{o} \rho \pi f_{r}}\right) r / r_{c}
$$

where $\mu$ is the permeability of free space, $\rho$ is the loop's material resistivity, $r$ is the radius of the loop, $r_{c}$ is the cross sectional wire radius, $\eta_{o}$ is the impedance of free space and $c$ is the speed of light. It should also be noted that (2)-(4) are valid only when $r<\lambda / 6 \pi$ [18]. The unloaded Q-factor can be written as :

$$
Q=\frac{\omega_{r} L}{R}=\frac{2 \pi f_{r} L}{R}
$$

The maximum Q-factor, $Q_{\max }$, and the frequency, $f_{\max }$, where $Q_{\max }$ occurs, derived from [19] as :

$$
\begin{aligned}
f_{\max }\left(r, r_{c}\right) & =\frac{c^{8 / 7} \mu^{1 / 7} \rho^{1 / 7}}{4 \cdot 15^{2 / 7} r_{c}^{2 / 7} \pi^{11 / 7} r^{6 / 7}} \\
Q_{\max }\left(r, r_{c}\right) & =\frac{2 \pi f_{\max } \mu_{0} r \ln \left(8 r / r_{c}-2\right)}{\left(\frac{\mu_{0} \rho \pi r^{2} f_{\max }}{r_{c}^{2}}\right)^{\frac{1}{2}}+20 \pi^{2}\left(\frac{2 \pi f_{\max } r}{c}\right)^{4}}
\end{aligned}
$$

In standard SCMR (1) to (7) are used to calculate the required optimal parameters, and can be modified to for three orthogonal loops in the orientation insensitive SCMR.

\section{MisALIGNMENT INSENSITIVE POWER TRANSFER}

In this section the similarity and differences between standard SCMR and two different misalignment insensitive SCMR model (2- and 3-loops structures) will be demonstrated with single turn loops. The specification of the model in Ansoft HFSS and laboratory measurement are : $r_{1}=50 \mathrm{~mm}, r_{2}$ $=45 \mathrm{~mm}, r_{3}=40 \mathrm{~mm}, r_{c}=2.2 \mathrm{~mm}, D=250 \mathrm{~mm}, f_{\max }=41.5$ $\mathrm{MHz}, Q_{\max }=740$ and the capacitor is $22 \mathrm{pF}, 56 \mathrm{pF}$ and $67 \mathrm{pf}$ in the 3-loops, 2-loops and standard SCMR system.

\section{A. Standard SCMR}

The comparison between the measurement and simulation is as shown in Fig. 4. In the models the Tx or RX and source

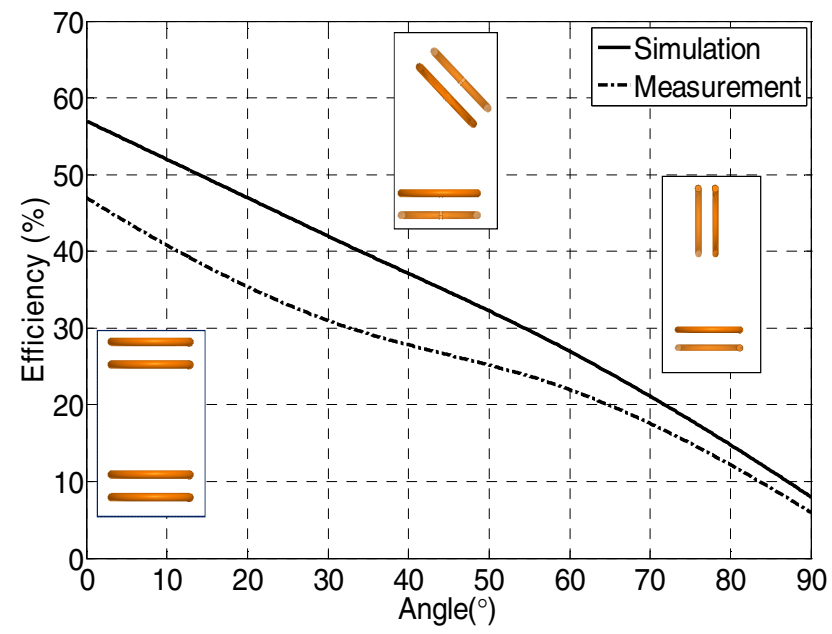

Fig. 4. SCMR with loops and angular elevation misalignment angles, $\theta=0^{\circ}$ to $90^{\circ}$.

elements (see Fig. 1) are fixed, while the other element are rotating as shown. The result showed that Fig. 4, the results 
show that as the angular misalignment angle, $\theta$ increases the efficiency drops, this is a major drawback for the standard SCMR method application in mobile devices. The angular azimuth and rotational misalignment performance are also poor.

\section{B. Misalignment Insensitive SCMR (2-loop structure)}

In the first orientation insensitive model designed (see Fig. 7 (a), it was observed that even though the model is efficient in with angular elevation and angular azimuth misalignment(see Fig. 5, however the efficiency significantly drops with rotational misalignment, the analysis (measurement and simulation) are carried out in a complete sphere, and the result of the rotational misalignment is as shown in Fig. 6. The efficiency drops significantly with rotational misalignment. In order to mitigate this low efficiency and achieve an SCMR system that is misalignment insensitive in the three directions, further modification are necessary to the model.

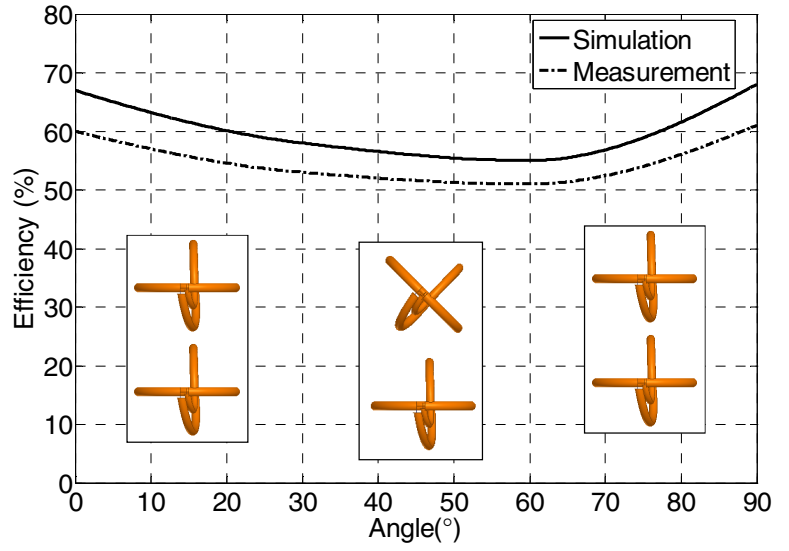

Fig. 5. SCMR with loops with angular elevation misalignment angles, $\theta=0^{\circ}$ to $90^{\circ}$.

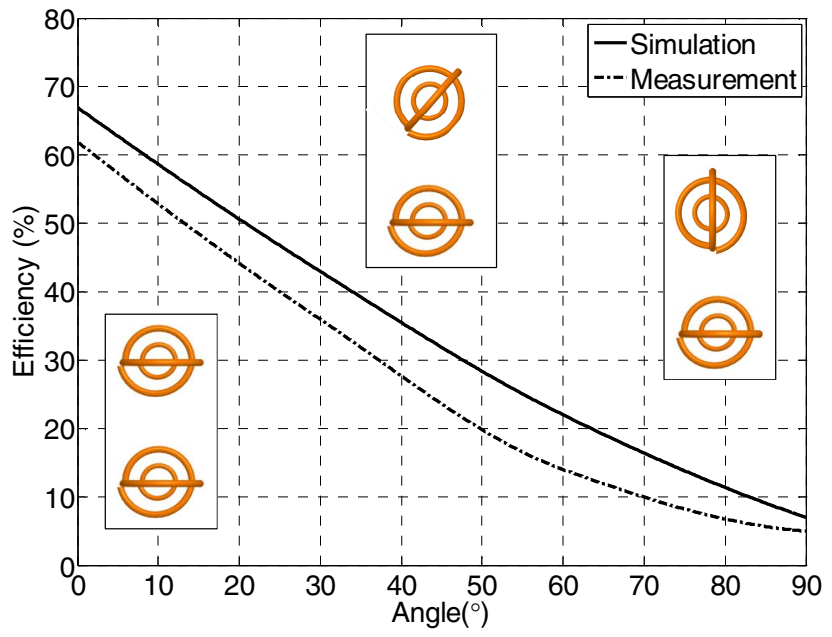

Fig. 6. SCMR with loops and rotational misalignment angles, $\alpha=0^{\circ}$ to $90^{\circ}$.

\section{Misalignment Insensitive SCMR (3-loop structure)}

Modification was carried out on the model of the Fig. 7(a), it was converted to 3D-loops (with three orthogonal loops each interconnected) and the source and load are embedded in the TX and RX elements as shown in Fig. 7(b).

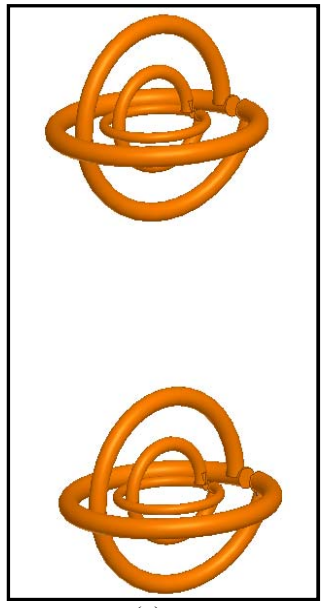

(a)

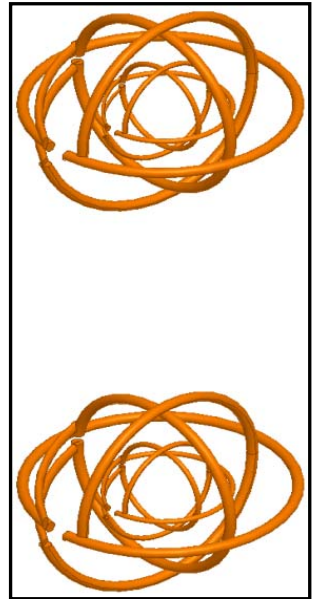

(b)
Fig. 7. SCMR structures with misalignment insensitivity. (a) SCMR with two orthogonal loops each (connected) and embedded. (b) SCMR with three orthogonal loops each (connected) and embedded.

The efficiencies plots of the Fig. 8 to Fig. 10 shows that 3loop misalignment insensitive structure will be efficient in these directions.

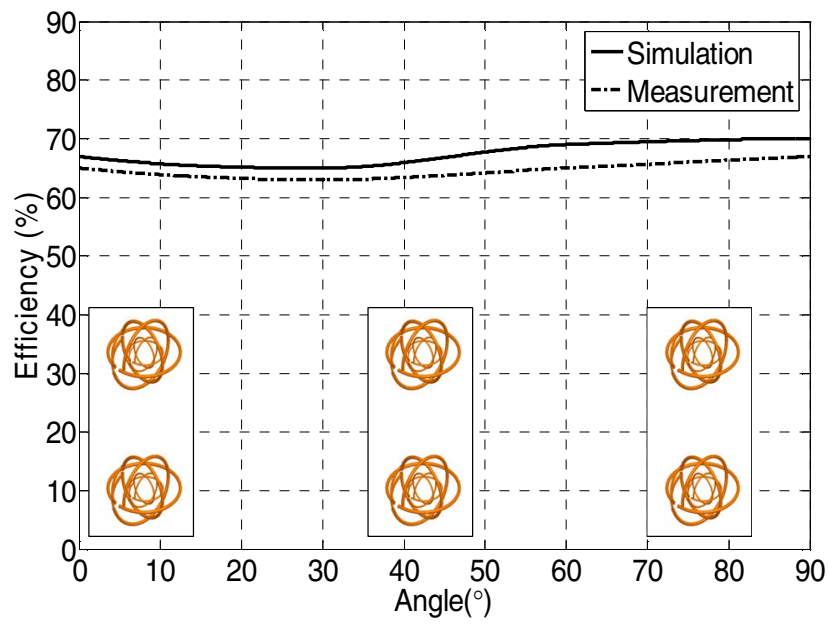

Fig. 8. SCMR with orthogonal (connected) loops, the source and load are embedded in the TX and RX devices with angular elevation misalignment $(\theta$ $=0^{\circ}$ to $90^{\circ}$ ).

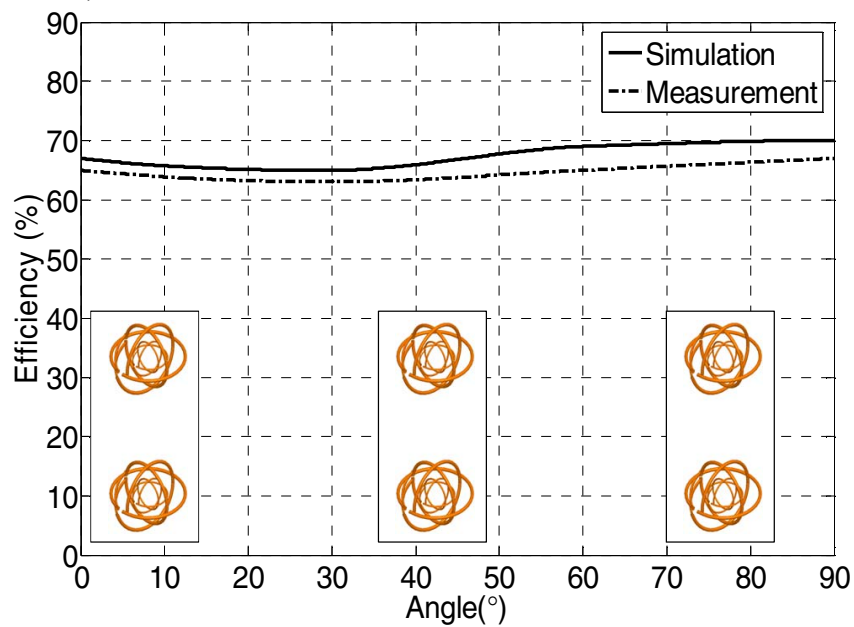

Fig. 9. SCMR with orthogonal (connected) loops, the source and load are embedded in the TX and RX devices with angular azimuth misalignment $(\varphi=$ $0^{\circ}$ to $90^{\circ}$ ). 


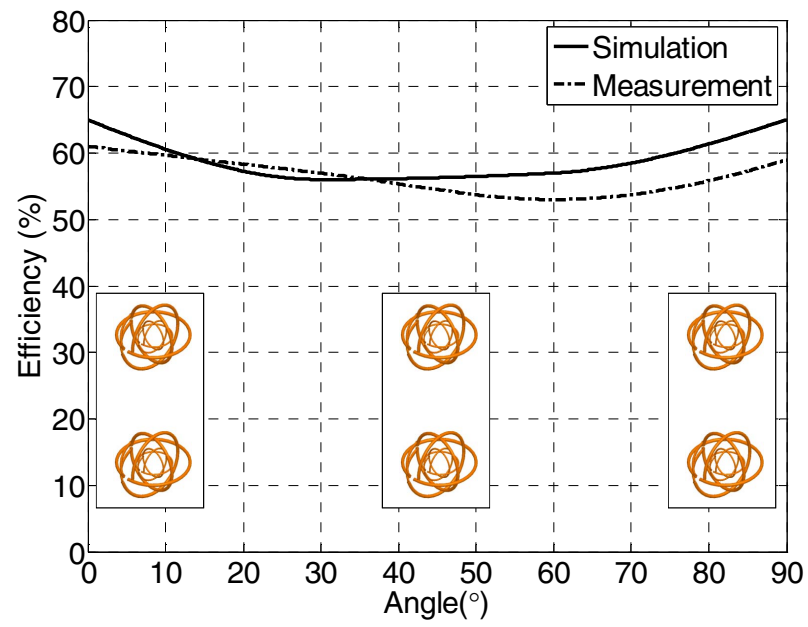

Fig. 10. SCMR with orthogonal (connected) loops, the source and load are embedded in the TX and RX devices with angular misalignment $\left(\alpha=0^{\circ}\right.$ to $90^{\circ}$ ).

The result of Fig. 4 to Fig. 10 shows that the result of the misalignment studies, it showed that only the 3-loop misalignment structure is efficient in all directions. The challenges of implementing this system can be solved by embedding the elements in the mobile device of interest. The system efficiency and range can be further improved by working with other materials and geometries.

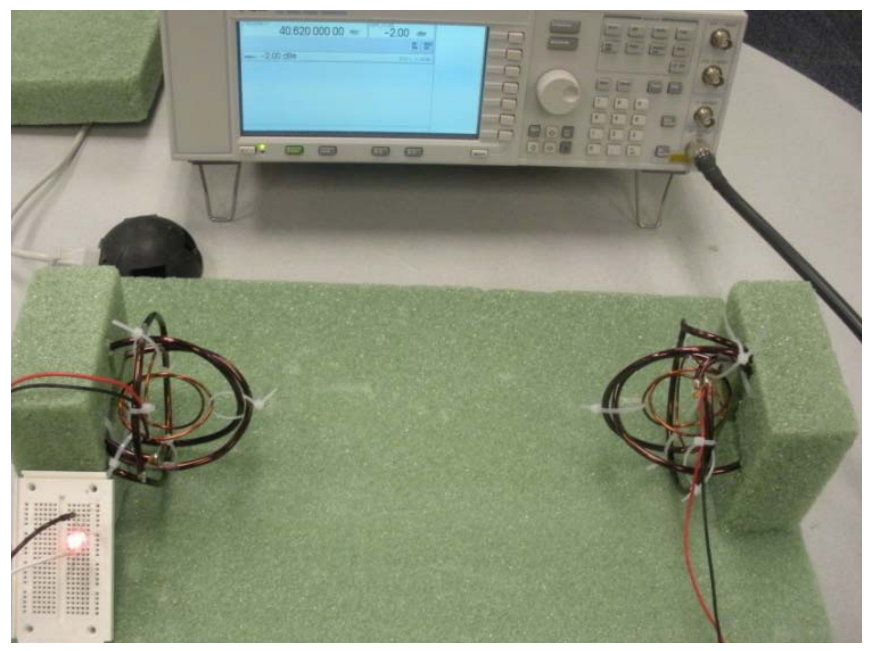

Fig. 11. Power transfer with embedded 3D loop (3- loop structure) in angular misalignment

\section{ACKNOWLEDGMENT}

This work was supported in part by the Dissertation Year Fellowship provided by Florida International University.

\section{CONCLUSIONS}

The paper presented optimized 3-loop misalignment structure for wireless power transfer via SCMR with misalignment insensitivity performance. The optimal SCMR features power transfer efficiencies above $60 \%$ over the misalignment range of $0^{\circ}-90^{\circ}$ degrees with angular, angular and lateral misalignment, which is much better than typical
SCMR elements utilized for wireless power transfer applications that suffer of efficiencies down to less than $10 \%$ in extreme misalignment topologies.

\section{REFERENCES}

[1] K. Finkenzeller, RFID Handbook: Fundamentals and Applications in Contactless Smart Cards and Identification, 2nd ed. New York:Wiley, 2003, pp. 65-112.

[2] P.V Nikitin, K.V.S Rao, S. Lazar , "An Overview of Near Field UHF RFID,” Proc. RFID IEEE Int. Conf., March 2007, pp. 167 - 174.

[3] G. Vandevoorde, and R. Puers, "Wireless energy transfer for standalone systems: a comparison between low and high energy applicability," Elsevier, Sensors and Actuators A: Physical, vol. 92, Issues 1-3, pp. 305-311, August 2001.

[4] C.A. Balanis, Antenna Theory: Analysis and Design, Wiley, New Jersey, 2005, ch. 5 .

[5] S. J. Mazlouman, A. Mahanfar, and B. Kaminska, "Mid-range wireless energy transfer using inductive resonance for wireless sensors", Proceedings of the IEEE international conference on Computer design. IEEE Press, Piscataway, NJ, USA, pp. 517-522, 2009

[6] A. Kurs, A. Karalis, R. Moffatt, J. D. Joannopoulos, P. Fisher, and M. Soljacic, "Wireless Energy Transfer via Strongly Coupled Magnetic Resonances," Science, vol. 317, pp. 83-85, 2007.

[7] A. Kurs, A. Karalis, R. Moffatt and M. Soljacic Marin, "Simultaneous midrange power transfer to multiple devices", Applied Physics Letter, vol. 96, 044102, 2010.

[8] A. Karalis, J.D. Joannopoulos, and M. Soljacic, "Efficient wireless nonradiative mid-range energy transfer," Elsevier Annals of Physics, vol. 323, pp. 34-48, January 2008.

[9] D. Joannopoulos, A. Karalis, and M. Soljacic, "Wireless Non- Radiative energy transfer," US Patent 20070222542, Sept. 2007.

[10] N.P. Cook, P. Meier, L. Sieber, M. Secall, H. Widmer, "Wireless Energy Apparatus and Method," US Patent 20080211320, Sept. 2008.

[11] B. L. Cannon, J. F. Hoburg, D.D. Stancil, S.C. Goldstein, "Magnetic Resonant Coupling As a Potential Means for Wireless Power Transfer to Multiple Small Receivers, "IEEE Transactions on Power Electronics, vol. 24, no.7, pp.1819-1825, July 2009.

[12] Olutola Jonah and S. V. Georgakopoulos, "Wireless Power Transmission to Sensors Embedded in Concrete via Magnetic Resonance", 2011 IEEE 12th Annual Wireless and Microwave Technology Conference, , vol., no., pp. 1-6, April 2011.

[13] S.G.Lee; H. Hoang, Y.H. Choi, F. Bien,; , "Efficiency improvement for magnetic resonance based wireless power transfer with axialmisalignment," Electronics Letters, Vol. 48, no. 6, pp. 339-340, March $15,2012$.

[14] K. Fotopoulou, B.W. Flynn, "Wireless Power Transfer in Loosely Coupled Links: Coil Misalignment Model," Magnetics, IEEE Transactions on Magnetics, vol.47, no.2, pp.416-430, Feb. 2011.

[15] M. A. Adeeb, A. B. Islam, M. R. Haider, F. S. Tulip, M. N. Ericson, and S. K. Islam, "An Inductive Link-Based Wireless Power Transfer System for Biomedical Applications," Active and Passive Electronic Components, vol. 2012, Article ID 879294, 11 pages, 2012.

[16] Wang Junhua; S.L Ho; W.N Fu; Sun Mingui; “Analytical Design Study of a Novel Witricity Charger With Lateral and Angular Misalignments for Efficient Wireless Energy Transmission, "IEEE Transactions on Magnetics, vol.47, no.10, pp.2616-2619, Oct. 2011.

[17] C. Reinhold, P. Scholz, W. John, and U. Hilleringmann, "Efficient Antenna Design of Inductive Coupled RFID-Systems with High Power Demand", Journal of Communications, vol. 2, no. 6, pp.14-23, November 2007.

[18] C.A. Balanis, Antenna Theory: Analysis and Design, Wiley, New Jersey, 2005.

[19] Olutola Jonah, Stavros V. Georgakopoulos and Manos M. Tentzeris, "Optimal Design Parameters for Wireless Power Transfer by Resonance Magnetic",IEEE Antennas and Wireless Propagation Letters, vol.11, no., pp.1390-1393, 2012 